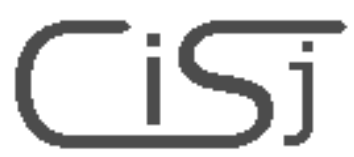

\title{
GROUP VELOCITY AND DISPERSION COEFFICIENT IN THE DISTRIBUTED SENSOR OF VIBRATION IN FIBRE OPTIC MICHELSON'S INTERFEROMETER CONFIGURATION
}

\author{
Bogdan Kizlik ${ }^{1)}$, Vitalij Nichoga ${ }^{2)}$, Petro Dub ${ }^{2)}$ \\ ${ }^{1)}$ Institute of Fundamental Electronics, Electronic Department, Military University of Technology, \\ 2 Kaliskiego Str., 00-908 Warsaw, Poland, \\ bkizlik@wel.wat.waw.pl \\ ${ }^{2)}$ Physico-Mechanical Institute of the National Academy of Sciences of Ukraine; \\ 5 Naukova St., 79601, Lviv, Ukraine; \\ nich@ah.ipm.lviv.ua
}

\begin{abstract}
The problem of determination of group velocity and dispersion coefficient in the distributed sensor of vibration in fibre optic Michelson's interferometer configuration is considered in the report. Influence of acoustic wave on optical wave in a Bragg modulator is described. It is shown that such interferometer can be used as a distributed highly sensitive sensor of mechanical values (vibration, acoustic wave, pressure, displacement).
\end{abstract}

Keywords: - Michelson's interferometer, fibre optic, distributed vibration sensor

\section{INTRODUCTION}

Interferometer as a classical technique is known for over 100 years. First research in this discipline were conducted by Michelson (1881), Mach Zehnder (1891), Fabry and Perot (1899) [1]. Fiber optic interferometer has been developing for the last ten years as a separate sort of fiber - optic sensors, functioning on a principle of optical wave phase changes detection. Optical configuration of interferometers has been adapted from classical interferometers where the light is lead in optical wave duct [2].

In scientific literature interferometric fiber - optics sensor is described as a device that processed of substitution of optical wave physical parameters for electric signal. Optic fibre sensor can be applied to detect a wide variety of external signals. The influence of mechanical (vibration, acoustic wave [3], pressure, displacement), magnetic [4], electric fields or temperature on light wave propagation can be easily detected event in closed optics circuit. Optic fibre interference sensors have extremely high sensitivity and closed optic circuit is their another advantage.

Optic fibre sensor consists of a light source, optic fibre and light detector, giving electric output signal. Optic fibre circuit has a sensing element - phase converter, which changes the phase of a light wave according to external signal. Those changes are converted back to electric signal in demodulator, which is an integral part of detector unit [5].

\section{WAVES IN THE FIBRE OPTIC MICHELSON'S INTERFEROMETER CONFIGURATION}

Single mode optic fibre carries coherent light wave, which has properties similar to a flat wave and is capable of interference. Distributed fibre optic sensor in Michelson interferometer layout is presented on Fig 1. If both arm of this interferometer are equal, it is called balanced one.

The phase distortion along the path L1 can be obtained on the basis of interference waves reflected from both arm of a sensor. Additional phase shift is introduced to achieve a clear distinction between consecutive wave packets.

If lengths of interferometer arms are different it is called unbalanced one. A Bragg cell (Fig 2) works as a light frequency shifter.

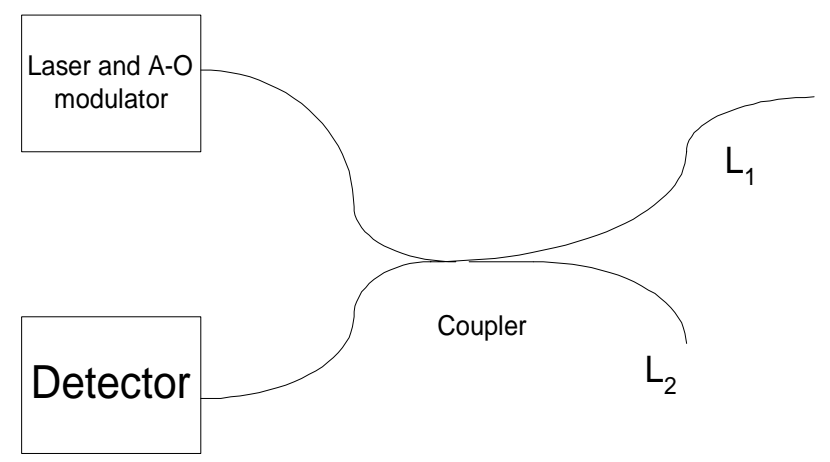

Fig. 1 - Unbalanced Michelson's interferometer as a distributed sensor. 
In Fig. 2:

$\Theta_{P}-$ angle of incidence of optical wave at Bragg modulator,

$\Theta_{P}-$ Bragg angle of refraction after passing the modulator,

$\omega_{i}-$ angular frequency of optical wave modulated by acoustic wave,

$\Omega_{i}$ - angular frequency of acoustic wave,

$L_{B}-$ Bragg modulator width,

$\mathbf{k}$ - optical wave vector,

$\lambda$ - optical wave length,

$\mathbf{K}$ - acoustic wave vector,
$\Lambda$ - acoustic wave length,

$\mathbf{V}_{a}$ - acoustic wave velocity,

$\mathbf{k}_{0}$ - optical wave that falls on a Bragg modulator,

$\mathbf{k}_{i}$ - optical wave after a Bragg modulator.

Interferometer is excited with wave packets shorter than their propagation time through the arm. The delay between packets $\tau$ is accordingly set to obtain interference in the coupler:

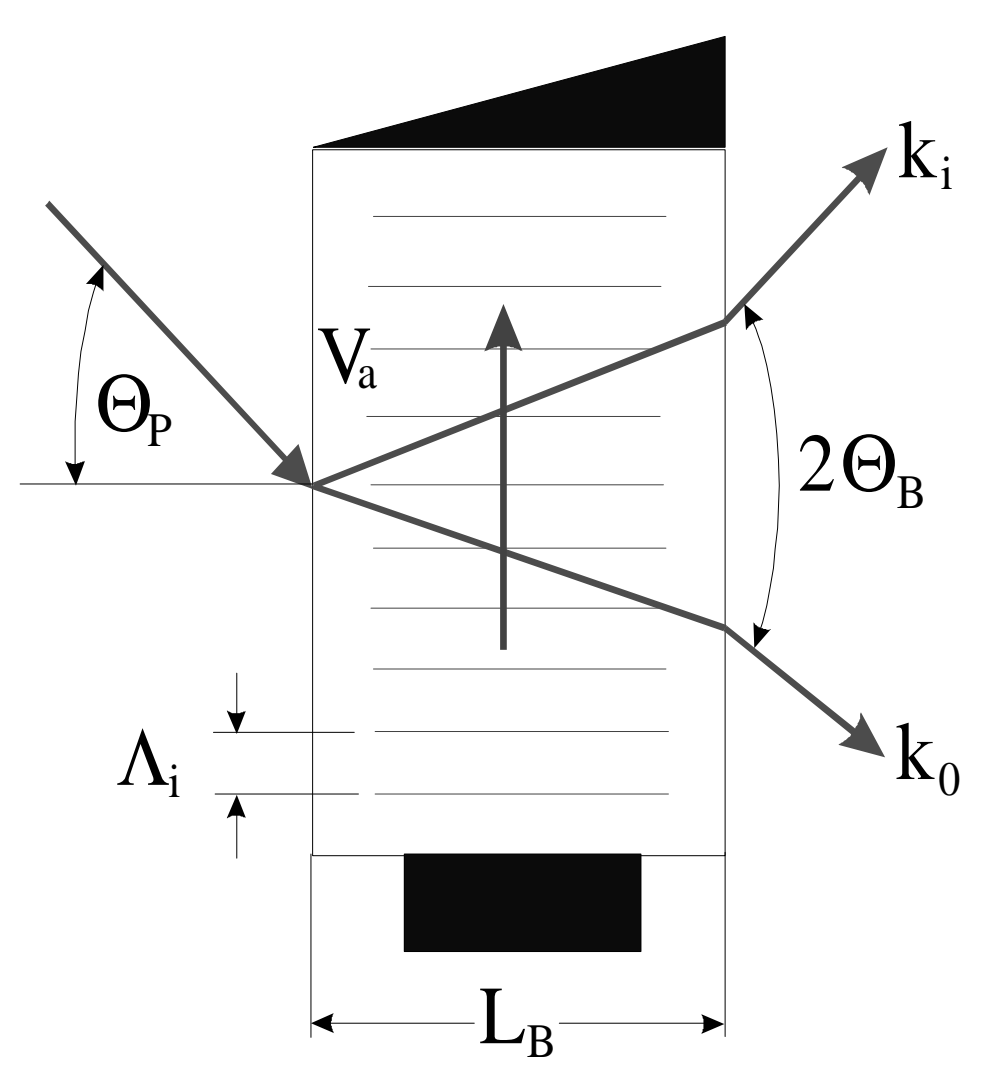

$$
\omega_{i}=\omega_{0}+\Omega_{i}
$$$$
\mathrm{k}_{\mathrm{i}}=\mathrm{k}_{0}+\mathrm{K}_{\mathrm{i}}
$$$$
\mathrm{K}_{\mathrm{i}}=2 \pi / \Lambda_{\mathrm{i}}
$$

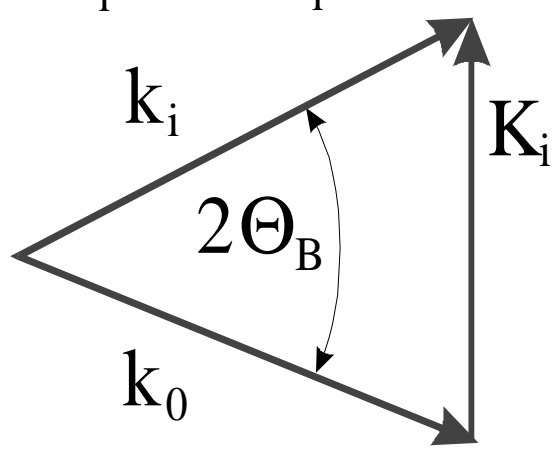

$$
\begin{aligned}
& \Theta_{\mathrm{B}}=\lambda / 2 \Lambda_{\mathrm{i}} \\
& \Lambda_{\mathrm{i}}=2 \pi \mathrm{V}_{\mathrm{a}} / \Omega_{\mathrm{i}}
\end{aligned}
$$

Fig 2 - Description of light frequency modulation with acoustooptic effect.

$$
\tau=\frac{2 L}{v}
$$

where

$L=L_{1}-L_{2}-$ difference of lengths of the interferometer arms;

$V$ - speed of light in optic fibre.

As a result, a signal proportional to phase difference is obtained, having $\Delta f=f_{1}-f_{2}$ frequency.

To simplify the analysis, polarization effects has been neglected which means, that constant polarization is assumed. Light wave at the output of frequency shifter (Fig. 2) with wave vector $\mathbf{k}_{i}=\mathbf{k}_{0}+\mathbf{K}_{i}$ and frequency $\omega_{\imath}=\omega_{0}+\Omega_{\mathrm{i}}$ can be described as

$$
E=\frac{1}{2} U_{B} \exp \left[j\left(\Phi+\omega_{i} t-\mathbf{k}_{i} \mathbf{r}\right)\right],
$$

where

$$
U_{B}=U_{p} \sin \frac{\Theta}{2},
$$

where

$U_{p}$ - amplitude of incident wave,

$\Theta$ - Bragg angle,

$\Phi$ - initial phase.

To simplify the description the following assumption were made: optic coupling Bragg cell fibre is lossless, wave is divided with 1:2 ratio and 
wave returning to coupler after passing measurement arm can be described as:

$$
E=\frac{1}{4} t_{m 1} U_{B} e^{j\left(\Phi+\omega_{1} t-\beta L_{1}\right)}
$$

where

$$
t_{m 1}-\text { transmission coefficient. }
$$

\section{GROUP VELOCITY AND DISPERSION COEFFICIENT IN DISTRIBUTED SENSOR OF VIBRATION}

A light wave modulated by a Bragg modulator assumes the following form (using its electrical vector):

$$
E=U_{B} \exp [j(\Phi+\omega t-\beta L)]
$$

where

$U_{B}$ - amplitude of the wave modulated in a Bragg modulator,

$\omega$ - angular frequency of light wave,

$\beta$ - propagation constant of light wave in light guide,

$L$ - light guide length.

Propagation constant of light wave in light guide can be presented as

$$
\beta=n(\mathbf{K}+\mathbf{k})(1-\Delta \mathrm{B})
$$

where

$n$ - index of refraction of light guide core,

$\mathbf{K}$ - wave vector of the acoustic wave,

$\mathbf{k}$ - wave vector of the light wave,

$\Delta, B$ - light guide parameters;

$\beta$ - light guide propagation constant expressed by the ratio

$$
\beta=\mathbf{n}_{1}|(\mathbf{K}+\mathbf{k})|\left(1-\Delta_{1} \mathbf{B}\right),
$$

where

$$
\begin{gathered}
\Delta_{1}=\frac{\mathbf{n}_{1}-\mathbf{n}_{2}}{\mathbf{n}_{1}}, \\
\mathbf{B}=\frac{\mathbf{n}_{1}^{2} \mathbf{k}_{0}^{2}-\beta^{2}}{\mathbf{n}_{1}^{2} \mathbf{k}_{0}^{2}-\mathbf{n}_{2}^{2} \mathbf{k}_{0}^{2}}=\frac{\mathbf{n}_{1}^{2}-\mathbf{n}_{e f}^{2}}{\mathbf{n}_{1}^{2}-\mathbf{n}_{2}^{2}}, \\
\mathbf{n}_{e f}=\frac{\beta}{\mathbf{k}_{0}} .
\end{gathered}
$$

Light wave falls at Bragg angle $\Theta$ at the acousticoptical modulator. Using Cartesian coordinate system to the modulator location in such way that the $Y$ axis is directed along acoustic wave propagation direction, the components of the two vectors in this system will have the following form:

$$
\mathbf{K}:[0, \mathrm{~K}] \text {, }
$$

$$
\mathbf{k}:\left[\mathrm{k}_{0} \cos (\Theta), \mathrm{k}_{0} \sin (\Theta)\right]
$$

Module of the stochastic vector is expressed by the following formula:

$$
\begin{aligned}
\left|\mathbf{K}_{\mathbf{w}}\right| & =\sqrt{\left[\mathbf{k}_{\mathbf{0}} \cos (\Theta)\right]^{2}+\left[\mathbf{K}+\mathbf{k}_{\mathbf{0}} \sin (\Theta)\right]^{2}}= \\
& =\sqrt{\left(\mathbf{k}_{\mathbf{0}}\right)^{2}+(\mathbf{K})^{2}+2 \mathbf{k}_{\mathbf{0}} \mathbf{K} \sin (\Theta)} ;
\end{aligned}
$$

from (8) follows that

$$
\left|\mathbf{K}_{\mathbf{w}}\right|=\mathbf{k}_{\mathbf{0}} \sqrt{1+\left(\frac{\mathbf{K}}{\mathbf{k}_{\mathbf{0}}}\right)^{2}+2 \frac{\mathbf{K}}{\mathbf{k}_{\mathbf{0}}} \sin (\Theta)}
$$

Expanding expression (9) into Taylor's series we receive in the first approximation:

$$
\left|\mathbf{K}_{\mathbf{w}}\right| \approx \mathbf{k}_{\mathbf{0}}\left[1+\frac{1}{2}\left(2 \frac{\mathbf{K}}{\mathbf{k}_{\mathbf{0}}} \sin (\Theta)+\left(\frac{\mathbf{K}}{\mathbf{k}_{\mathbf{0}}}\right)^{2} \cos ^{2}(\Theta)\right)+\ldots\right]
$$

Substituting expression (10) into (6), the formula for propagation constant of light wave modulated acoustically wave can be obtained:

$$
\begin{aligned}
& \beta \approx \mathbf{n k}_{0}\left[1+\frac{1}{2}\left(2 \frac{\mathbf{K}}{\mathbf{k}_{\mathbf{0}}} \sin (\Theta)+\right.\right. \\
& \left.\left.+\left(\frac{\mathbf{K}}{\mathbf{k}_{\mathbf{0}}}\right)^{2} \cos ^{2}(\Theta)\right)\right](1-\Delta \mathbf{B}) .
\end{aligned}
$$

Wave vectors depend on wave length:

$$
\mathbf{k}_{0}=\frac{2 \pi}{\lambda_{0}}
$$

for light wave with wave length $\lambda_{0}$,

$$
\mathbf{K}=\frac{2 \pi}{\Lambda}
$$

for light wave with wave length $\Lambda$, and

$$
\begin{aligned}
& \mathbf{c}=\lambda_{0} v_{0}, \\
& \mathbf{v}=\Lambda \mathbf{f},
\end{aligned}
$$




$$
\begin{gathered}
\mathbf{k}_{0}=\frac{2 \pi v_{0}}{\mathbf{c}}, \\
\mathbf{K}=\frac{2 \pi \mathbf{f}}{\mathbf{v}},
\end{gathered}
$$

$v-$ acoustic wave velocity,

$$
\begin{gathered}
\beta \approx\left[\mathbf{n} \mathbf{k}_{0}+\frac{1}{2} 2 \frac{2 \pi \mathbf{n}}{\mathbf{v}} \mathbf{f} \sin (\Theta)+\right. \\
\left.+\frac{1}{2} \mathbf{f}^{2} \frac{2 \pi \mathbf{n c}}{v_{0} \mathbf{v}^{2}} \cos ^{2}(\Theta)\right](1-\Delta \mathbf{B}), \\
\beta \approx 2 \pi \mathbf{n}\left[\frac{v_{0}}{\mathbf{c}}+\mathbf{f} \frac{\sin (\Theta)}{\mathbf{v}}+\frac{1}{2} \mathbf{f}^{2} \frac{\mathbf{c} \cos ^{2}(\Theta)}{v_{0} \mathbf{v}^{2}}\right](1-\Delta \mathbf{B})
\end{gathered}
$$

Assuming that dependency of propagation constant $\beta\left(v_{0}+f\right)$ expanded into Taylor's series is expressed by

$$
\beta\left(v_{0}+f\right)=\beta\left(v_{0}\right)+f \frac{d \beta}{d v}+\frac{1}{2} f^{2} \frac{d^{2} \beta}{d v^{2}},
$$

group velocity is equal to

$$
\begin{gathered}
\frac{1}{v_{g r}}=\frac{1}{2 \pi} \frac{d \beta}{d v}, \\
\frac{1}{\mathbf{v}_{\mathbf{g r}}}=\frac{n \sin (\Theta)}{\mathbf{v}}(1-\Delta \mathbf{B}),
\end{gathered}
$$

and dispersion coefficient equals

$$
\begin{gathered}
D_{v}=\frac{1}{2 \pi} \frac{d^{2} \beta}{d v^{2}}, \\
\mathbf{D}_{v}=\frac{\mathbf{n c} \cos ^{2}(\Theta)}{v_{0} \mathbf{v}^{2}}(1-\Delta \mathbf{B}) .
\end{gathered}
$$

\section{CONCLUSIONS}

\section{Group Velocity}

If the dispersion coefficient is sufficiently small, the third term in the expansion (13) may be neglected and

$$
E(f)=E(0) \exp \left(-j 2 \pi f \tau_{d}\right)
$$

The system is then equivalent to an attenuation factor and a time delay

$$
\tau_{d}=L / v_{g},
$$

so that

$$
E(z, t)=\exp (-\alpha L / 2) E\left(0, t-\tau_{d}\right)
$$

In this approximation the pulse travels at the group velocity (14) $v_{g}$, its intensity is attenuated by the factor $\exp (-\alpha L / 2)$, but its initial shape is not altered. By comparison, in an ideal (lossless and nondissipated) medium, $\alpha=0$ and $\beta(v)=2 \pi v / c$, so that $v_{g}=c$ the pulse envelope travels at the speed of light in the medium and its height and shape are not altered.

\section{Dispersion Coefficient}

Since the group velocity

$$
v_{g r}=2 \pi /(d \beta / d v)
$$

is itself frequency dependent, different frequency component of the pulse undergo different delays

$$
\tau_{d}=L / v_{g r}
$$

As a result, the pulse spreads and its shape is altered.

Two identical pulses of central frequencies $v$ and $v+\delta v$ suffer a differential delay:

$$
\delta \tau=\frac{d \tau_{d}}{d v} \delta v=\frac{d}{d v}\left(\frac{L}{v}\right) \delta v=D_{v} L \delta v
$$

If $D_{v}>0$ (normal dispersion), the travel time for the higher - frequency component is longer than the travel time for the lower - frequency component. Thus shorter - wave length components are slower. Normal dispersion occurs in glass in the visible band.

If the pulse has a spectral width $\sigma_{v}(\mathrm{~Hz})$ then:

$$
\sigma_{\tau}=\left|D_{v}\right| \sigma_{v} L
$$

is an estimate of the spread of its temporal width. The dispersion coefficient $D_{v}$ is therefore a measure of the pulse time broadening per unit spectral width per unit distance $(\mathrm{s} / \mathrm{m} \cdot \mathrm{Hz})$.

$$
E(0)=\exp (-\alpha z / 2)
$$




\section{REFERENCES}

[1] T. Okosi, K. Okomoto, M. Otsu and others. FibreOptic Sensors. (Russian translation). Energoizdat. Leningrad, 1990. p. 250.

[2] M. Szustakowski, W. Ciurapinski, L. Jodlowski. Some Issues on Phase Analysis, Molecular and Quantum Acoustics 20 (1999) pp. 263277.

[3] M. Chojnacki, B. Kizlik, W. Ciurapinski. Distributed Sensor of Vibration in Fibre Optic Michelson's Interferometer Configuration. Proceedings of the VI-th International Conference the Experience of Designing and Application of CAD Systems in Microelectronics (CADSM'2001), Lviv-Slavsko, Ukraine 12-17 February 2001, pp. $183-186$.

[4] V. Nichoga, G. Trokhym. On a Possibility of Usage of Optic Fibre Sensors in Infralow-frequency Geomagnetic Measurements and Requirements to Them, (in Russian), Geofizicheskaya Apparatura 94 (1991). pp. 13-20.

[5] V.N.Mahajan Theory of acoustooptic interaction standing and traveling sound waves, Wave Electronics 2 (1976). pp. $309-339$.

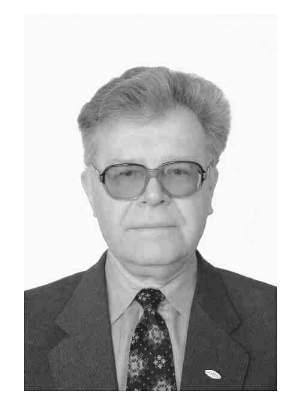

Vitaliy Nichoga was born in Kiev in 1938. graduated radiotechnical faculty of National University "Lviv polytechnics" in 1960. He has obtained PhD degree in electrical and radiotechnical measurements in 1966. Since 1968 he is senior researcher. He has obtained post-doctoral degree in 1996 in Kiev institute of control and liaison. He works in physical and mechanical institute of National academy of sciences of Ukraine by G.V.Karpenko since 1960 as researcher. Now he is a leading researcher of division of acquisition and processing of stochastic signals. At the same time Vitaliy Nichoga is a professor of department of radioelectronical devices and systems of radiotechnical faculty of national University "Lviv Polytechnics" and associated editor of scientific journal "Radioelectronics and telecommunications", which is published by this university.

Main scientific interests of Vitaliy Nichoga: methods and means of magnetic quantities measurements, designing of mathematical models of electromagnetic fields, creation on the bases of new physicals phenomena, technologies and magnetic materials of high efficient mobile antennas and sensors for measuring of low electromagnetic fields, which are used in such areas as diagnosis and nondestructive control, geophysics and space explorations, information defense in telecommunication systems. Vitaliy Nichoga is the author of more than 190 scientific papers, including two monographs; he has obtained 10 inventors certificates and patents.

Petro Dub was born in Lviv in 1954. In 1977 he graduated faculty of automatics at national university "Lviv polytechnics". Since that time he woks as engineer and senior engineer in physical and mechanical institute of National academy of sciences of Ukraine by G.V.Karpenko.

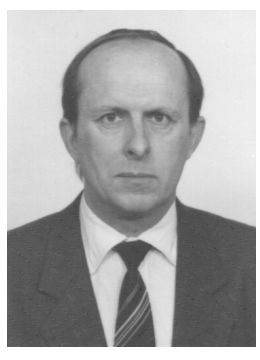

His main scientific interests are learning of electromagnetic fields and designing of tools for their measurement. He is the author of 75 scientific papers. 